\title{
Dynamics of the recuperative mechanical stepless vehicle's drive
}

\author{
Sergey Hoodorozhkov ${ }^{1}$ and Aleksandr Kozlenok $^{1 *}$ \\ ${ }^{I}$ Peter the Great St. Petersburg Polytechnic University, Polytechnicheskaya, 29, , St. Petersburg, \\ 195251, Russia. E-mail: Kozlenok.A.V@gmail.com
}

\begin{abstract}
The article represents the results of a theoretical study into the operation of a mechanical stepless vehicle drive with the recuperation of braking energy and flywheel energy storage. This paper mainly focused on Adjustable-Speed Drive (ASD) as a part of the infinite ratio continuously variable transmission equipped with flywheel energy storage. To study the proposed transmission, the authors compiled a mathematical model in the form of a system of nonlinear differential equations. The proposed model allows you to determine and monitor the change in the angular velocities and angular accelerations of the rotating links of the transmission. The results of the work will be used to analyze the components of a stepless drive in order to optimize its components to reduce the response time and increase the transmission efficiency, which will lead to a reduction in mechanical losses during the movement of the proposed vehicle.
\end{abstract}

\section{Introduction}

The existing mechanical stepless transmissions are usually friction-type: V-belt, disc or ball-and-socket gears. These variators belong to the category of CVT type (continuously variable transmission) stepless transmissions. Their main drawback is a small range of torque transformation (2...5), the need to use an automatic clutch to start from standstill and the lack of self-regulation of the gear ratio at load changes. For these reasons, the inclusion of such gears in the regenerative movement systems of vehicles is impractical.

The mechanical CVTs based on the use of freewheel clutches manufactured by ZeroMax Drives (USA) belong to the category of Adjustable-Speed Drive (ASD) variators. These are stepless transmissions of Infinitely Variable Transmission (IVT) type, in which, unlike the CVT, the possibility of continuous gear ratio variability is implemented not within a limited but within an "infinitely" wide range of output shaft rotation speeds, including the stop mode.

The main control principle of the ASD variator is a stepless speed control of the output shaft by changing the amplitude of the oscillations of four or more roller freewheel clutches, which alternately rotate the output shaft. The variator has high efficiency $(\eta>0.92)$, simple design, is easy to adjust and maintain, and it is also cheaper than the other types. The main disadvantage is the lack of self-regulation of the gear ratio depending on the

* Corresponding author: Kozlenok.A.V@gmail.com 
external load.

To eliminate this disadvantage and use an ASD variator in regenerative drives, the transmission is supplemented by elastic linkage, for example, a torsion shaft, which allows to carry out automatic torque transformation with a constant amplitude of oscillations of the driving unit of the freewheel clutch by changing the torsion bar twist angle, providing an automatic increase in torque (decrease in the speed of the driven shaft) with an increase in load and, conversely, a smooth decrease in torque (increase in the rotation speed of the output shaft) with a decrease in load $[1,2]$.

Fig. 1 shows a basic kinematic diagram of a recuperative mechanical system consisting of two identical flywheels connected by two identical but contra directional kinematic chains [3].

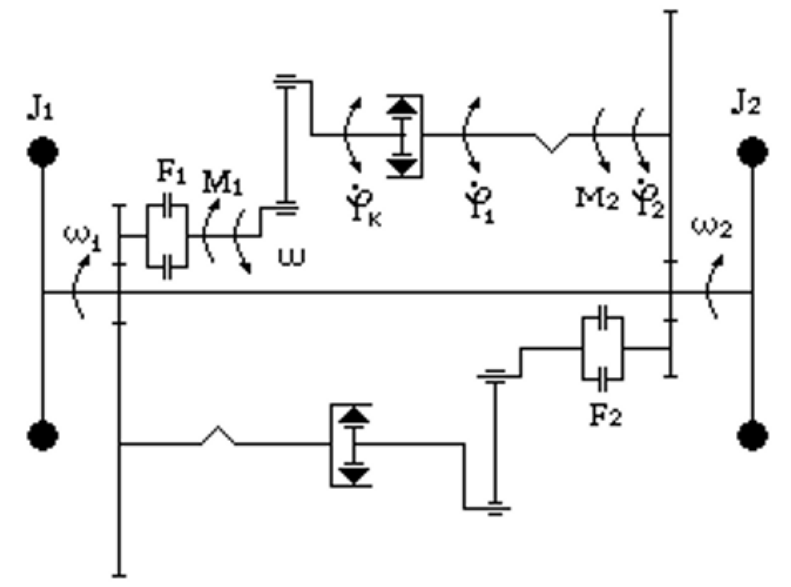

Fig. 1. A recuperative mechanical system

Each chain has a drive clutch, an articulation four-link chain, a freewheel clutch, a torsion shaft and a transmission with a constant gear ratio. Such a kinematic chain has the properties of a torque transformer.

\section{Materials and methods}

Let us consider the process of energy exchange between the flywheels when $F_{l}$ clutch is involved. At the initial moment of time, the $J_{l}$ flywheel has an angular velocity of $\omega_{01}=\omega_{\max }$, while $J_{2}$ flywheel has $\omega_{02}=\omega_{\text {min }}$. The $J_{1}$ flywheel decelerates, and the $J_{2}$ flywheel accelerates. At the end of braking/acceleration, it has to be $\omega_{1}=\omega_{\text {min }}$, and $\omega_{2}=\omega_{\max }$. Then, when the $F_{1}$ clutch is turned off and the $F_{2}$ clutch is turned on, the process will be repeated in exactly the same manner, but in the opposite direction. In the work [4], the following ratio was produced in relation to this kinematic scheme:

$$
\left\{\begin{array}{l}
\omega_{\max }=\varphi_{0} \cdot i_{\max } \cdot I_{r_{-} \text {in }} \cdot I_{r_{-} \text {out }} \cdot \omega_{\min } \\
\omega_{\min }=\varphi_{0} \cdot i_{\min } \cdot I_{r_{-} \text {in }} \cdot I_{r_{-} \text {out }} \cdot \omega_{\max } .
\end{array}\right.
$$

Here, $i_{\min }$ and $i_{\max }$ are the minimum and maximum values of the gear ratio, which can be changed by twisting of the torsion shaft from 0 when the output shaft is stationary (stop mode) to 1 in the absence of a load on it; $\varphi_{0}$ is the amplitude of the oscillations of the freewheel clutch driver; $I_{r_{-} \text {in }}, I_{r_{-} \text {out }}$ is the constant reduction ratio in each kinematic chain. 
On the basis of (1), we have

or

$$
\frac{\omega_{\max }}{\omega_{\min }}=\varphi_{0} \cdot i_{\max } \cdot I_{r_{-} \text {in }} \cdot I_{r_{-} \text {out }}=\frac{1}{\varphi_{0} \cdot i_{\min } \cdot I_{r_{-} \text {in }} \cdot I_{r_{-} \text {out }}}
$$

$$
\varphi_{0}^{2} \cdot I_{r_{-} \text {in }}^{2} \cdot I_{r_{-} \text {out }}^{2} \cdot i_{\max } \cdot i_{\min }=1
$$

Dependence (3) is an expression of the circular gear ratio of the circuit when both clutches are engaged, $\left(F_{l}\right.$ and $\left.F_{2}\right)$. In fact, only one of them is involved at a given moment. If we set the value of $\varphi_{0}$ (the design parameter) and $i_{\max }$ and $i_{\min }$ values, the dependence (3) will determine the required value of $I_{r_{-} \text {in }}, I_{r_{-} \text {out }}$ and the range value of the flywheel's angular velocities variation.

Thus, dependence (3) establishes a relationship between the value of the selected range $D$ of the $i$ change in the gear ratio

$$
D_{i}^{2}=\operatorname{imin}_{\max } \text { and } D=\sqrt{D_{i}}
$$

and the values $\varphi_{0}$ and the constant gear ratio of the matching redactors $I_{r_{-} \text {in }}$, $I_{r_{\text {out }} \text {. This }}$ makes it possible to carry out kinematic calculation of the regenerative drive (to determine the transfer ratios on the input and output of IVT transmission with the freewheel clutch). An example of plotting the change in the angular velocities of the flywheels with the involvement of $F_{1}$ and $F_{2}$ clutches is performed in the work [4] on the basis of the study of the recuperative system of a $260-\mathrm{kg}$ bike car, equipped with a $10-\mathrm{kg}$ flywheel and a recuperative mechanical stepless drive (Fig. 1).

It is practical to start the theoretical study of the internal dynamics of the working processes of a recuperative mechanical stepless transmission with the consideration of the simplest three-mass model (with elasticity at the output of the freewheel clutch) shown in Fig. 2 (the general case with the external input torque $M_{d}$ ). The model in Fig. 2, at the top, corresponds to the period of the effective run of the transmission (joint movement of the driving and driven parts of the transmission), and Fig. 2, at the bottom, to the idle period (the driving and driven systems move independently).

When constructing a mathematical model, it should be borne in mind that it is practical to solve dynamics problems with a nonlinear position function, such as the case with this gear, with the use of a special type of Lagrange equations with "extra" coordinates.

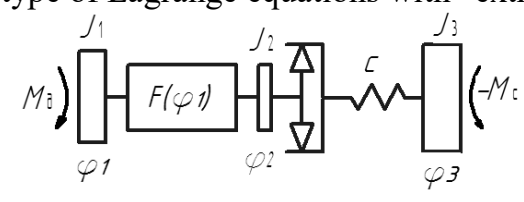

Working stroke

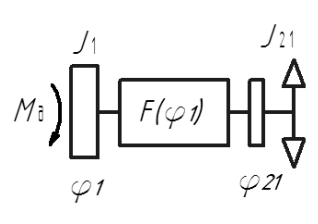

Driving

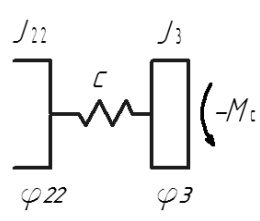

Driven

Idle stroke

Fig. 2. The three-mass model of mechanical stepless transmission at the stages of effective and idle running 
Let us construct the equations of motion for the transmission model during its effective running (Fig. 2, above) with respect to the second derivatives of the generalized coordinates:

Lagrange equations with "extra" coordinates are as follows:

$$
\frac{d}{d t}\left(\frac{\partial T}{\partial \dot{\phi}_{j}}\right)-\frac{\partial T}{\partial \phi_{j}}+\frac{\partial V}{\partial \phi_{j}}=Q_{j}+\sum_{i=1}^{n} \lambda_{i} \cdot h_{i j}
$$

Here $\lambda_{\mathrm{i}}$ is the Lagrange multiplier, and $h_{i j}$ is the coefficient with the Lagrange multiplier. Generalized coordinates selection:

$$
\varphi_{2}=F\left(\varphi_{1}\right)
$$

Here $\varphi_{1}, \varphi_{2}, \varphi_{3}$ are the angular movement coordinates of the corresponding masses, and $F\left(\varphi_{1}\right)$ is the function of the position.

Then the expression of kinetic energy through generalized velocities will be equal to:

$$
\begin{gathered}
T=\frac{1}{2} \cdot\left[J_{1} \cdot \dot{\phi}_{1}{ }^{2}+J_{2} \cdot \dot{\phi}_{2}{ }^{2}+J_{3} \cdot \dot{\phi}_{3}{ }^{2}\right] ; \\
\frac{\partial T}{\partial \dot{\phi}_{1}}=J_{1} \cdot \dot{\phi}_{1} ; \frac{\partial T}{\partial \dot{\phi}_{2}}=J_{2} \cdot \dot{\phi}_{2} ; \frac{\partial T}{\partial \dot{\phi}_{3}}=J_{3} \cdot \dot{\phi}_{3} ; \\
\frac{d}{d t}\left(\frac{\partial T}{\partial \dot{\phi}_{1}}\right)=J_{1} \cdot \ddot{\phi}_{1} ; \frac{d}{d t}\left(\frac{\partial T}{\partial \dot{\phi}_{2}}\right)=J_{2} \cdot \ddot{\phi}_{2} ; \frac{d}{d t}\left(\frac{\partial T}{\partial \dot{\phi}_{3}}\right)=J_{3} \cdot \ddot{\phi}_{3} ; \\
\frac{\partial T}{\partial \phi_{1}}=\frac{\partial T}{\partial \phi_{2}}=\frac{\partial T}{\partial \phi_{3}}=0 ;
\end{gathered}
$$

The expression of potential energy in generalized coordinates, including the "extra" ones:

$$
\begin{gathered}
V=\frac{1}{2} \cdot\left[c \cdot\left(\phi_{2}-\phi_{3}\right)^{2}\right]=\frac{1}{2} \cdot\left[c \cdot\left(\phi_{2}^{2}-2 \cdot \phi_{2} \cdot \phi_{3}+\phi_{3}^{2}\right)^{2}\right] \\
\frac{\partial V}{\partial \phi_{1}}=0 ; \frac{\partial V}{\partial \phi_{2}}=c \cdot \phi_{2}-c \cdot \phi_{3} ; \frac{\partial V}{\partial \phi_{3}}=c \cdot \phi_{3}-c \cdot \phi_{2}
\end{gathered}
$$

The equation relating the "extra" coordinate to the independent generalized coordinate:

$$
\varphi_{2}=F\left(\varphi_{1}\right)
$$

Let us differentiate the last equation with respect to time and write it as follows:

Then

$$
\dot{\phi}_{2}=F^{\prime}\left(\phi_{1}\right) \cdot \dot{\phi}_{1} ; \ddot{\phi}_{2}=F^{\prime \prime}\left(\phi_{1}\right) \cdot \dot{\phi}_{1}^{2}+F^{\prime}\left(\phi_{1}\right) \cdot \ddot{\phi}_{1} ;
$$

$$
F^{\prime}\left(\phi_{1}\right) \cdot \dot{\phi}_{1}-\phi_{2}=0
$$

Here $F^{\prime}\left(\varphi_{1}\right)=\frac{d F}{d \varphi_{1}}$ is the transfer function.

The constraint equation is as follows:

$$
h_{1} \cdot \dot{\phi}_{1}+h_{2} \cdot \dot{\phi}_{2}+h_{3} \cdot \dot{\phi}_{3}+h=0
$$

Comparing (15) with (16), we write down $h_{1}=F^{\prime}\left(\phi_{1}\right) ; h_{3}=-1$.The remaining coefficients are zero.

Summarized forces determination. By producing an expression of the sum of the works of non-potential forces on possible movements and grouping the members at $\delta q_{i}$, we define $Q_{i}$. 


$$
Q_{i}=\sum_{1}^{3} M_{i} \cdot h_{i} Q_{1}=M_{\partial} ; Q_{2}=-M_{c} ; Q_{3}=-M_{c}
$$

As a result, the system of differential equations is as follows:

$$
\left\{\begin{array}{l}
J_{1} \cdot \ddot{\phi}_{1}=M_{\partial}+\lambda \cdot F^{\prime}\left(\phi_{1}\right) ; \\
J_{2} \cdot \ddot{\phi}_{2}=-c \cdot\left(\phi_{2}-\phi_{3}\right)-\lambda ; \\
J_{3} \cdot \ddot{\phi}_{3}=c \cdot\left(\phi_{2}-\phi_{3}\right)-M_{c} ;
\end{array}\right.
$$

We use the second equation to determine the Lagrange multiplier $\lambda$ :

$$
\lambda=-J_{2} \cdot \ddot{\phi}_{2}-c \cdot\left(\phi_{2}-\phi_{3}\right) ;
$$

Given the fact that

we produce

$$
\dot{\phi}_{2}=F^{\prime}\left(\phi_{1}\right) \cdot \dot{\phi}_{1} ; \ddot{\phi}_{2}=F^{\prime \prime}\left(\phi_{1}\right) \cdot \dot{\phi}_{1}^{2}+F^{\prime}\left(\phi_{1}\right) \cdot \ddot{\phi}_{1} ;
$$

$$
\lambda=-c \cdot\left(\phi_{2}-\phi_{3}\right)-J_{2} \cdot\left[F^{\prime \prime}\left(\phi_{1}\right) \cdot \dot{\phi}_{1}^{2}+F^{\prime}\left(\phi_{1}\right) \cdot \ddot{\phi}_{1}\right] ;
$$

Then, by substituting $\lambda$ into the system (12), we have

$$
\left\{\begin{array}{l}
\ddot{\phi}_{1}=\left\{M_{\partial}+F^{\prime}\left(\phi_{1}\right) \cdot\left[-C_{1} \cdot\left(\phi_{2}-\phi_{3}\right)-J_{2} \cdot \dot{\phi}_{1}{ }^{2} \cdot F^{\prime \prime}\left(\phi_{1}\right)\right]\right\} /\left(J_{1}+J_{2} \cdot F^{\prime}\left(\phi_{1}\right)^{2}\right) ; \\
\ddot{\phi}_{2}=\dot{\phi}_{1}{ }^{2} \cdot F^{\prime \prime}\left(\phi_{1}\right)+\ddot{\phi}_{1} \cdot F^{\prime}\left(\phi_{1}\right) \\
\ddot{\phi}_{3}=\left[C_{1} \cdot\left(\phi_{2}-\phi_{3}\right)-M c\right] / J_{3}
\end{array}\right.
$$

Here $F\left(\phi_{1}\right)=\phi_{0} \cdot\left(1-\cos \phi_{1}\right) ; F^{\prime}\left(\phi_{1}\right)=\phi_{0} \cdot \sin \left(\phi_{1}\right) ; F^{\prime \prime}\left(\phi_{1}\right)=\phi_{0} \cdot \cos \left(\phi_{1}\right)$ is the position function (gearing ratio), its 1 st (gearing function) and 2 nd derivatives, respectively; $M_{d}$ and $M_{s}$ are the reduced moments of the engine and the resistance; $C$ is the normalized coefficients of the roll rate; $J_{1,2,3}$ are the reduced moments of inertia of the masses.

At the idle stage, the system falls into two independent subsystems, the dynamic models of which are shown in Fig. 2 below.

The equations describing the behavior of the driving subsystem in the idling period with respect to the second-order derivatives have the form of (23), while the equations describing the behavior of the driven subsystem have the form of (24)

$$
\begin{aligned}
& \left\{\begin{array}{l}
\left.\ddot{\phi}_{1}=\left\{M_{\partial}+F^{\prime}\left(\phi_{1}\right) \cdot\left[-J_{21} \cdot \dot{\phi}_{1}{ }^{2} \cdot F^{\prime \prime}\left(\phi_{1}\right)\right]\right\} / J_{1}+J_{21} \cdot F^{\prime}\left(\phi_{1}\right)^{2}\right) ; \\
\ddot{\phi}_{21}=\dot{\phi}_{1}{ }^{2} \cdot F^{\prime \prime}\left(\phi_{1}\right)+\ddot{\phi}_{1} \cdot F^{\prime}\left(\phi_{1}\right) ;
\end{array}\right. \\
& \left\{\begin{array}{l}
\ddot{\phi}_{22}=-C_{1} \cdot\left(\phi_{22}-\phi_{3}\right) / J_{22} ; \\
\ddot{\phi}_{3}=\left[C_{1} \cdot\left(\phi_{22}-\phi_{3}\right)-M_{c}\right] / J_{3} .
\end{array}\right.
\end{aligned}
$$

Here, $J_{21}, J_{22}$ are the inertia moments of mass 2, reduced to the driver and the driven unit of the freewheel clutch; $\varphi_{21}$ and $\varphi_{22}$ are the coordinates of masses with inertia moments $J_{2 l}$ and $J_{22}$. We will take the values of inertial elastic coefficients for the scheme in Fig. 4 as based on the reduction of the inertial-elastic units of the bike car [2] without taking into account the friction losses in the chain drive and in the freewheel clutch. 


\section{Results}

Obtained nonlinear differential equations describe the operation of a stepless transmission as a mechanical system with a variable structure on the basis of one of the numerical methods give us follow results. Conditions of the bike car movement: - acceleration from zero initial speed (the angular velocity of the bike car normalized by the output shaft $\left.\omega_{B}^{n p}=0\right)$;

$f=0.012$ is the travel resistance coefficient;

- the reduced driving torque is zero $M_{d}=0$ (acceleration with flywheel);

-the moment of resistance brought to the output shaft at regenerative acceleration, $\mathrm{Nm}$

$$
M_{c_{\_} n p}=16.37
$$

- the moment of inertia of the flywheel normalized to the input shaft of the stepless transmission is equal to, $\mathrm{kg}^{*} \mathrm{~m}^{2}$

$$
J_{M}^{n p}=0.646
$$

- the maximum angular velocity of the flywheel normalized by the input shaft, radian/s

$$
\omega_{M}^{n p_{-} \max }=111.7
$$

- the moment of inertia of the bike car reduced to the output shaft of the stepless transmission, $\mathrm{kg}^{*} \mathrm{~m}^{2}-J_{B}^{n p}=74.3$

Fig. 3,4 and 5 shows the fragments of working processes in the mechanical stepless transmission of a bike car constructed on the numerical computation of the equation system of $14,15,16$. The graphs are produced for the normalized angular velocities of inertial masses $J_{21}, J_{22}, J_{3}$ at the stages of effective and idle running of the freewheel clutch for the entire period of recuperative acceleration of the bike car (Fig. 3), in the initial (Fig. 4) and final Fig. 5) periods of acceleration.

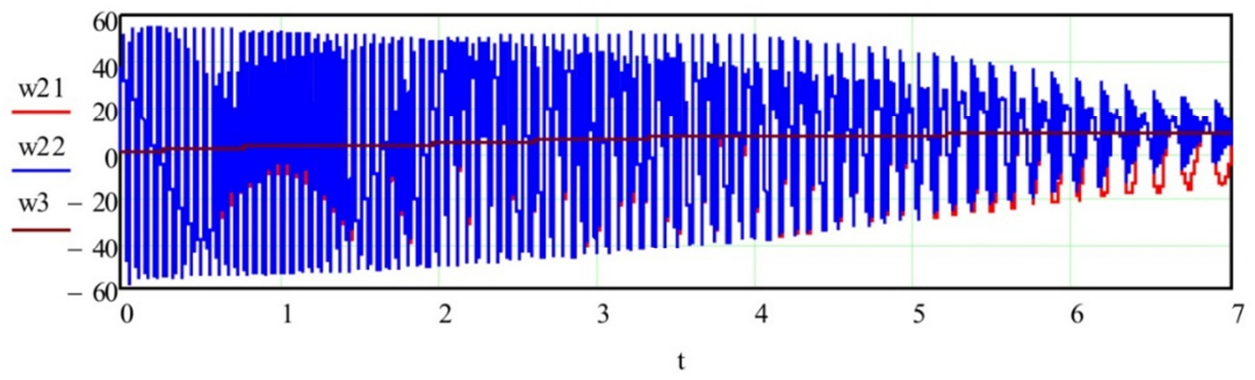

Fig. 3. The graphs of the changes in the normalized angular velocities of transmission linkage $\omega_{21}$, $\omega_{22}, \omega_{3}$ (radian/second) during acceleration $t$, for the entire period of the bicycle car recuperative acceleration

From the graphs, it follows that at the beginning of acceleration there is a maximum amplitude of the reduced angular velocity of the flywheel, $\omega_{21}(55 \mathrm{rad} / \mathrm{sec})$ and the minimum phase of idle running, approximately $0.005 \mathrm{sec}$. At the end of acceleration, the amplitude of the specific angular velocity of the flywheel decreases to $25 \mathrm{radian} / \mathrm{s}$, and the idle phase increases to 0.05 seconds. This corresponds to an increase in the linear speed of the bike car from zero to $17 \mathrm{~km} / \mathrm{h}$ and a decrease in the speed of the flywheel from 2030 rpm to $500 \mathrm{rpm}[5]$. 


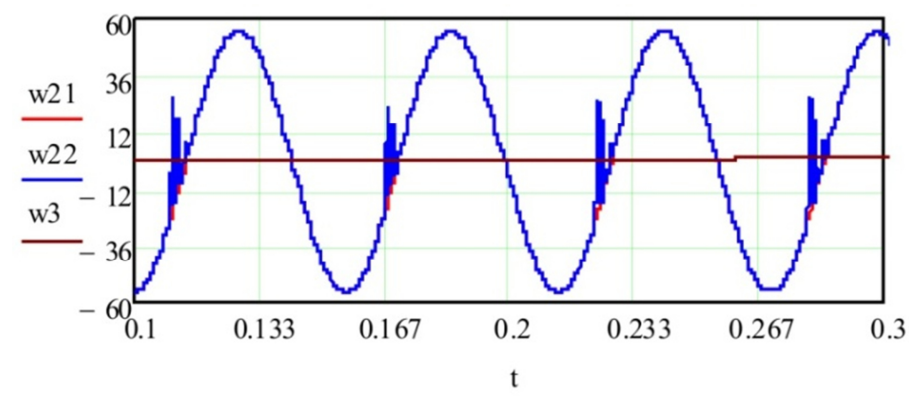

Fig. 4. The graphs of the changes in the normalized angular velocities of transmission linkage $\omega_{21}$, $\omega_{22}, \omega_{3}$ (radian/second) during acceleration $t$, in the initial period of the bicycle car recuperative acceleration

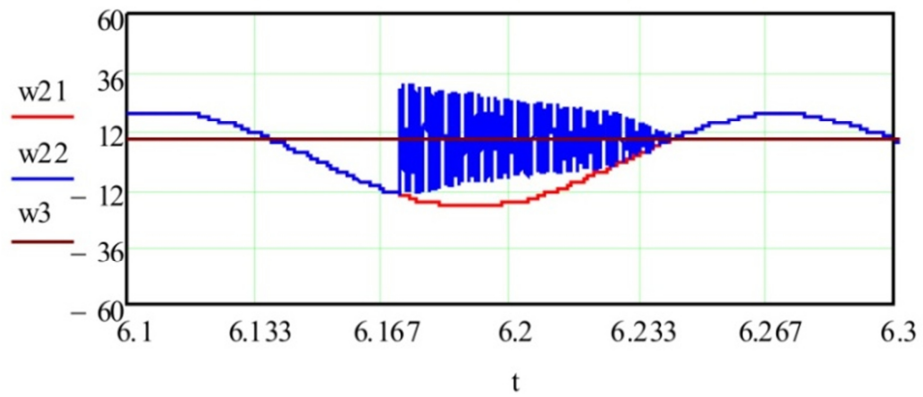

Fig. 5. The graphs of the changes in the normalized angular velocities of transmission linkage $\omega_{21}$, $\omega_{22}, \omega_{3}$ (radian/second) during acceleration $t$, in the final period of the bicycle car recuperative acceleration

The nature of the torque $\left(\mathrm{N}^{*} \mathrm{~m}\right)$ changes at the input and output of the stepless transmission is shown in Fig. 6, 7and 8:

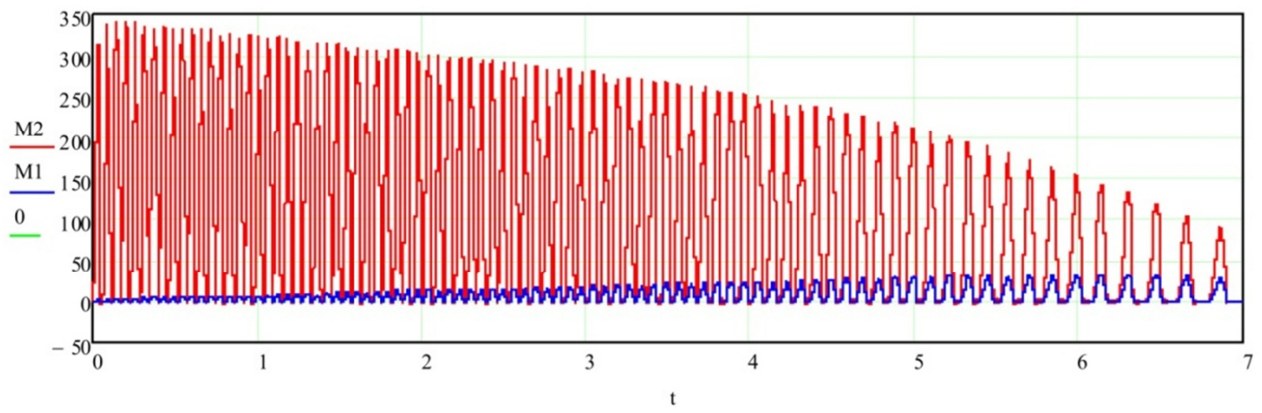

Fig. 6. The graphs of torque change on input $\mathrm{M}_{1}$ and output $\mathrm{M}_{2}$ transmission shafts during the entire period of the bicycle car recuperative acceleration 


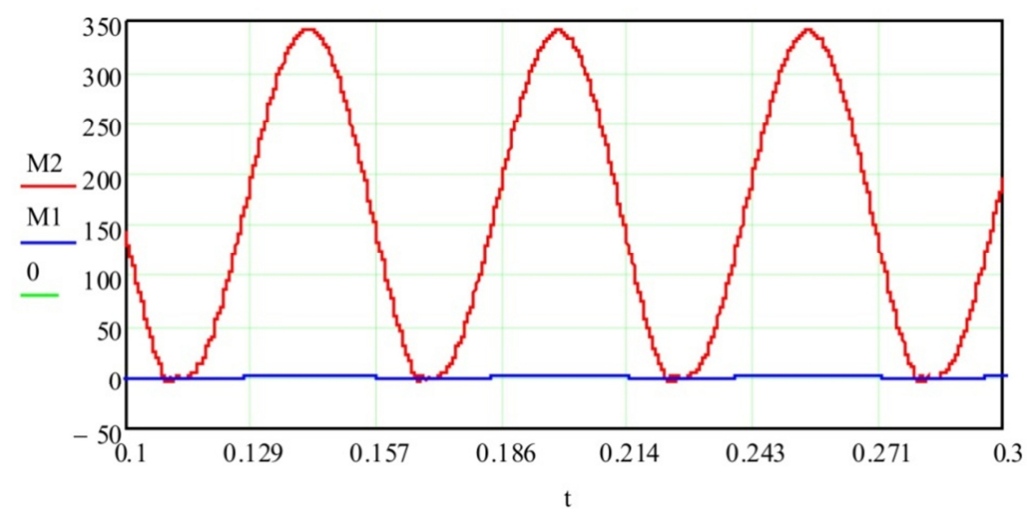

Fig. 7. The graphs of torque change on input $\mathrm{M}_{1}$ and output $\mathrm{M}_{2}$ transmission shafts during the initial period of the bicycle car recuperative acceleration

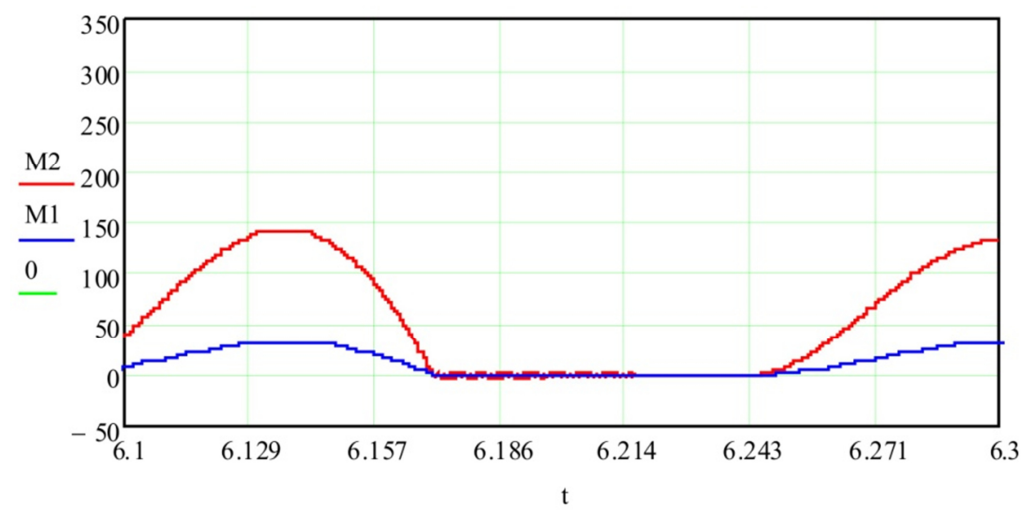

Fig. 8. The graphs of torque change on input $\mathrm{M}_{1}$ and output $\mathrm{M}_{2}$ transmission shafts during the final period of the bicycle car recuperative acceleration

\section{Conclusion and discussion}

The analysis of the graphs shows that with an increase in speed from $0 \mathrm{~km} / \mathrm{h}$ to $17 \mathrm{~km} / \mathrm{h}$, the maximum torque on the output shaft is automatically reduced from 345 to $90 \mathrm{~N} * \mathrm{~m}$. The torque transformation coefficient in the transmission changes with the increase of the output shaft speed from nearly 30 (at the start from standstill) to 3.6 at the maximum speed. The cycle time of the transmission work process increases from 0.057 to 0.2 seconds.

The maximum values of linear acceleration during acceleration occur in the initial period and do not exceed $2 \mathrm{~m} / \mathrm{s}^{2}$, decreasing to $0.3 \mathrm{~m} / \mathrm{s}^{2}$ at the end of acceleration.

Due to the symmetry of the circuits, the dynamic processes in a stepless transmission with regenerative braking are similar to the process of regenerative acceleration, and, accordingly, the working processes are similar to those shown in Figures 3 and 4.

The obtained results of the theoretical study into the dynamics of the working processes in the recuperative mechanical stepless transmission allow us to conclude that the developed mathematical model and the methods of its research are effective. The performed calculations confirm the effectiveness of this direction of technical development in the field of mechanical stepless transmissions of vehicles with the recovery of braking energy $[5,6]$. 


\section{References}

1. S. Hoodorozhkov, A. Krasilnikov. SHS Web of Conf., 44, 7-14 (2018). DOI: 10.1051/shsconf/20184400038.

2. S. Hoodorozhkov. MATEC Web of Conferen $\sqsubset$ es, 245, 10-20 (2018).DOI:10.1051/matecconf/201824507012

3. S. Hoodorozhkov., St. Petersburg Polytechnic University Journal of Engineering Sciences and Technology, 3(249), 118-127 (2016). DOI: 10.5862/JEST.249.14

4. M. Hedlund, J. Lundin. Energies, 8(10), 10636-10663 (2015).DOI:10.3390/en81010636.

5. S. Mohammad, F. Faraji, A. Majazi, K. Al-Haddad. Renewable and Sustainable Energy Reviews, 67, 477-490. DOI: 10.1016/j.rser.2016.09.060

6. K. Itani, A. De Bernardinis, Z. Khatir, A. Jammal. Energy Conversion and Management, 144, 69-87 (2017).DOI: 10.1016/j.enconman.2017.04.036

7. K. Berkel, S. Rullens, T. Hofman, B. Vroemen, M. Steinbuch. IEEE Transa tions on Vehi ular Te hnology, 63, 4192-4205 (2014). DOI: 10.1109/TVT.2014.2312646

8. B. Wang, D. Hung, J. Zhong, K. The. International Journal of Automotive Technology, 19, 907-914 (2018). DOI: 10.1007/s12239-018-0087-z

9. T. Yukawa. Renewable Energy and Power Quality Journal, 9, 130-135 (2011). DOI:10.24084/repqj09.264

10. T. Yukawa, A. Murakami, Y. Sato, S. Ohshima. Journal of the Japan Society for Precision Engineering, 79, 943-949 (2013). DOI: 10.2493/jjspe.79.943

11. T. Yukawa, S. Kumagai, T. Fujisawa, Y. Oshida, Y. Takeda. Mechanical Engineering Research, 9, 20-27 (2019). DOI:10.5539/mer.v9n2p20

12. A. Soomro, M. Amiryar, K. Pullen, D. Nankoo. Energy Pro edia, 151, 100-110 (2018). DOI:10.1016/j.egypro.2018.09.034

13. F. Morales, F. Benitez. Mechanism and Machine Theory, 134, 197-212 (2019). DOI: 10.1016/j.mechmachtheory.2018.12.030

14. N. Srivastava, I. Haque. Mechanism and Machine Theory, 44, 19-41 (2009). DOI: 10.1016/j.mechmachtheory.2008.06.007

15. Y. Mingyao, Q. Datong, Z. Xingyu, Z. Sen, Z. Yuping. Mechanism and Machine Theory, 136, 52-71 (2019). DOI: 10.1016/j.mechmachtheory.2019.02.014 